\title{
Determining Medium Range Atomic Ordering in Metallic Glasses Using 4D-STEM
}

Soohyun Im $^{1}$, Pengyang Zhao ${ }^{2}$, Geunhee Yoo $^{3}$, Zhen Chen ${ }^{4}$, Gabriel Calderon Ortiz ${ }^{1}$, Mehrdad Abbasi Gharacheh $^{1}$, Olivia Licata ${ }^{5}$, Baishakhi Mazumder ${ }^{5}$, David Muller ${ }^{4}$, Eun Soo Park ${ }^{3}$, Yunzhi Wang ${ }^{1}$ and Jinwoo Hwang ${ }^{1}$

${ }^{1}$ The Ohio State University, Columbus, Ohio, United States, ${ }^{2}$ Shanghai Jiao Tong University, Shanghai, Shanghai, China (People's Republic), ${ }^{3}$ Seoul National University, Seoul, Seoul-t'ukpyolsi, Republic of Korea, ${ }^{4}$ Cornell University, Ithaca, New York, United States, ${ }^{5}$ University at Buffalo-SUNY, Buffalo, New York, United States

Medium range atomic ordering (MRO) attributes to nanometer scale structural heterogeneity in metallic glasses (MGs), and it has been under extensive interest due to its potential connection to the important properties of the material, including mechanical properties and glass forming ability. However, the precise characterization of MRO has been challenging. Conventional large area diffraction methods, such as using $\mathrm{X}$-ray, neutron, or electron, inherently average the structure within the illuminated volume, through which the MRO information tend to be lost. MRO domains in MGs is typically smaller and much less ordered as compared to nanocrystals, and therefore observing them using high-resolution TEM is also very difficult. The difficulties have made the understanding of the exact relationship between MRO and important properties of MGs very challenging. For example, it has been speculated that MRO may be related to the mechanical properties of MGs (e.g. [2]), especially their ductility, but no clear experimental evidence for this suggested relationship has been shown so far. MRO may also be related to the glass forming ability of MGs [3], although spatially resolved information of MRO must be required to confirm the hypothesis.

In this work, we determine the structural parameters of MRO with unprecedented details using 4dimensional scanning transmission electron microscopy (4D-STEM) [4, 5]. Our 4D-STEM is enabled by the quantitative analysis of the data acquired using the new-generation Electron Microscopy Pixel Array Detector (EMPAD) [6], which provides high dynamic range essential for the quantification. About 250,000 nanodiffraction patterns were acquired per sample using STEM probes with $1 \mathrm{~nm}$ in diameter, continuously throughout the sample area with over sampling (Fig. 1a). The resulting 4D data were then reconstructed into dark field images in the real space for all scattering vector magnitude, $k$, and the inplane azimuthal angle of (Fig. $1 \mathrm{~b}$ and 1c). These images show bright speckles of the MRO domains, which we use to quantitatively determine the MRO parameters, including their type, size, distribution, and volume fraction.

The determined MRO parameters are shown to be directly related to the important properties of the $\mathrm{Zr}$ $\mathrm{Cu}-\mathrm{Co}-\mathrm{Al}$ MGs. The data shows that the smaller and more diverse MRO types (Fig. 1d) lead to higher ductility. Certain MRO types are more structurally frustrated, as revealed by the angular correlation function analysis of the 4D-data (Fig. 2a), which correlates well with the glass forming ability of the MGs. To understand the mechanism of the observed correlation, we have used mesoscale deformation simulation directly incorporating the MRO data determined by 4D-STEM (Fig. 2b). The simulation shows the changes in the deformation behavior, including the full development of shear bands and predictive quantities for materials' failure, which confirms the correlation between the MRO and properties observed experimentally [7]. 
(a) Probe
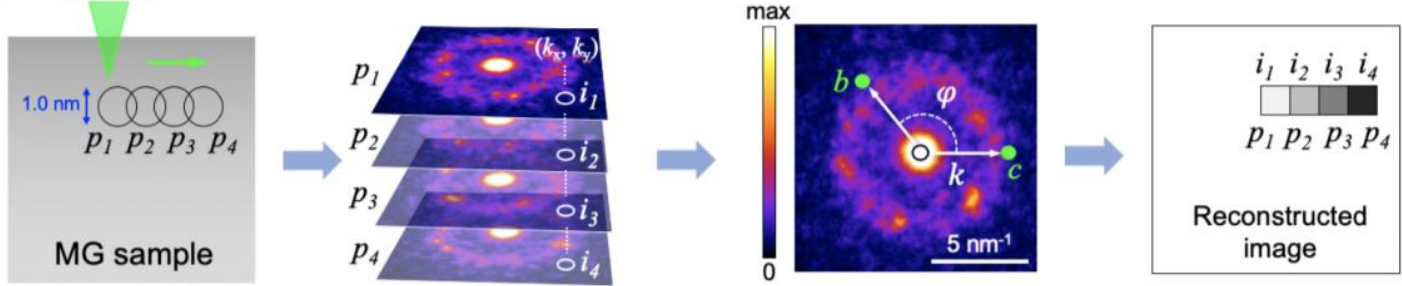

(b)

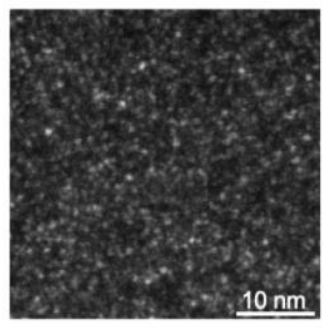

(c)
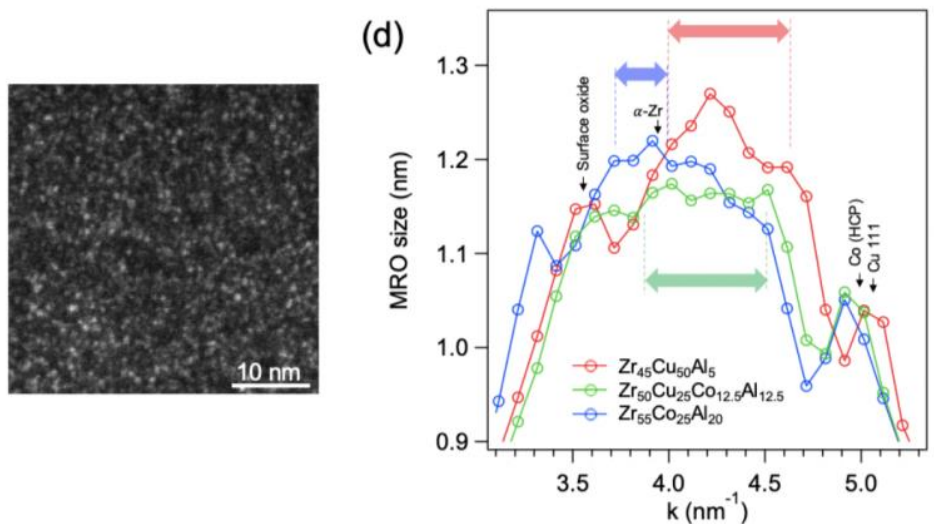

Figure 1. Figure 1. (a) Schematic of 4D-STEM. Nanodiffraction patterns are acquired using electron probe $($ diameter $=1 \mathrm{~nm})$ from oversampled probe positions $(\mathrm{p} 1, \mathrm{p} 2, .$.$) on the sample. The intensities (i1,$ $\mathrm{i} 2, .$.$) in the acquired stack of patterns can then be reconstructed in the real space by selecting any (kx, ky)$ pixel within the pattern. (b and c) The reconstructed "dark-field" using the "b" and "c" pixels in (a), respectively. (d) Average MRO size calculated from the 4D data.
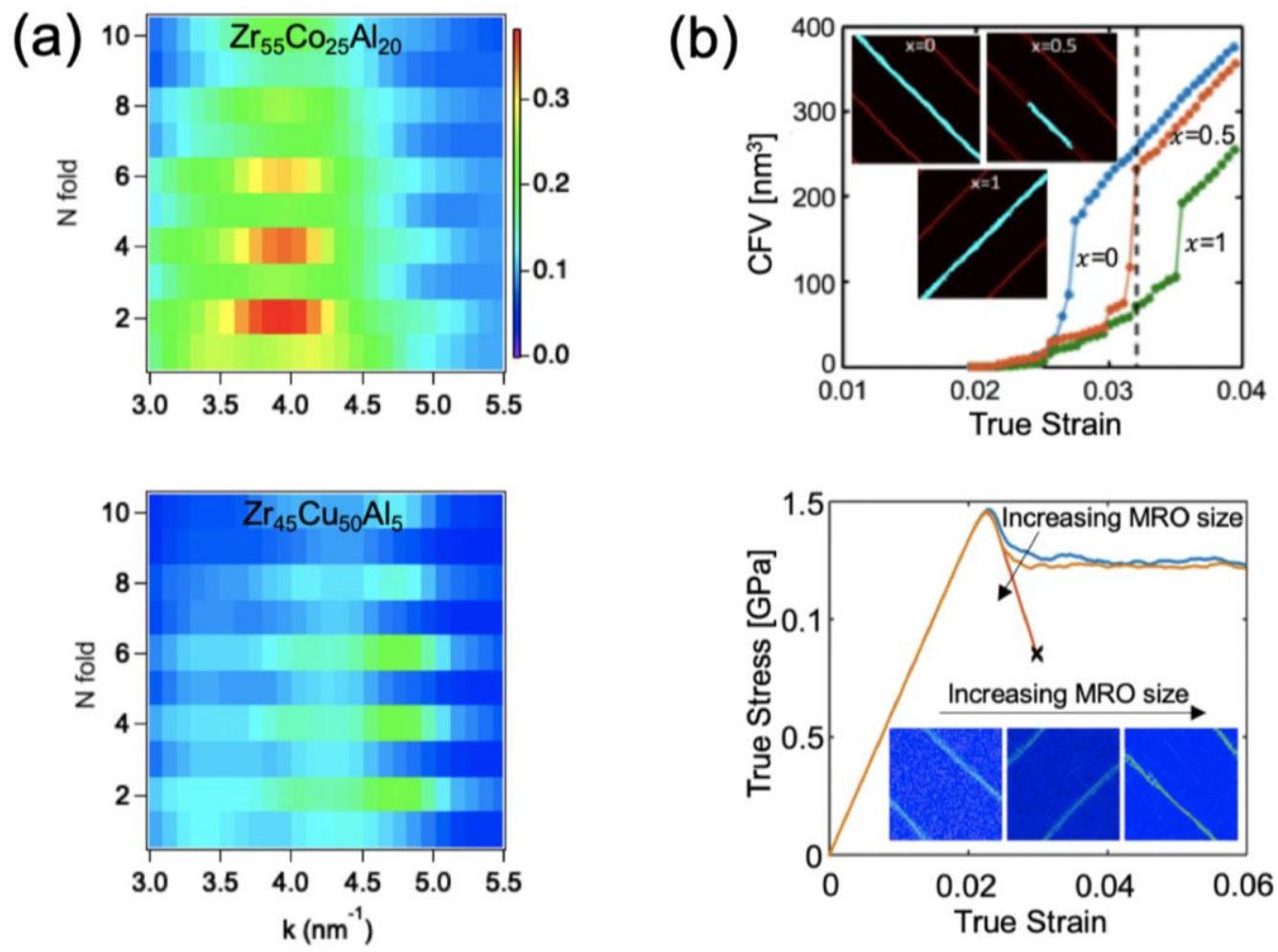
Figure 2. Figure 2. (a) Angular correlation determined from 4D-STEM data showing different types and degree of MRO in different MGs. (b) Mesoscale deformation simulation results confirming the effect of MRO to mechanical properties.

\section{References}

[1] J. Hwang et al., Physical Review Letters, 108(19), 195505 (2012).

[2] A.S. Argon, Acta Metallurgica, 27(1), 47-58 (1979).

[3] C. E. Pueblo, M. Sun, and K. F. Kelton, Nat. Mater. 16, 792 (2017).

[4] S. Im, Z. Chen, J. M. Johnson, P. Zhao, G. H. Yoo, E. S. Park, Y. Wang, D. A. Muller, and J. Hwang, Ultramicroscopy 198, 189 (2018).

[5] Soohyun Im, Pengyang Zhao, Geun Hee Yoo, Zhen Chen, Gabriel Calderon, Mehrdad Abbasi Gharacheh, Olivia Licata, Baishakhi Mazumder, David A. Muller, Eun Soo Park, Yunzhi Wang, and Jinwoo Hwang, Physical Review Letters (in review).

[6] M. W. Tate, P. Purohit, D. Chamberlain, K. X. Nguyen, R. Hovden, C. S. Chang, P. Deb, E. Turgut, J. T. Heron, D. G. Schlom, D. C. Ralph, G. D. Fuchs, K. S. Shanks, H. T. Philipp, D. A. Muller, and S. M. Gruner, Microsc. Microanal. 22, 237 (2016).

[7] This work is supported by NSF under DMR-1709290, and it was performed in part at the Cornell PARADIM Electron Microcopy Facility, as part of the Materials for Innovation Platform Program, which is supported by the NSF grant under DMR-1539918 with additional infrastructure support from DMR1719875 and DMR-1429155. 